\title{
Digastric Muscle
}

National Cancer Institute

\section{Source}

National Cancer Institute. Digastric Muscle. NCI Thesaurus. Code C32462.

A small, striated muscle located under the mandible. It arises from the mastoid process and extends to the symphysis menti, and functions to lower the mandible and elevation of the hyoid bone. 